\title{
THE APPROXIMATE DIVERGENCE OPERATOR
}

VICTOR L. SHAPIRO ${ }^{1}$

1. We shall operate in Euclidean $k$-space, $k \geqq 2$, and shall denote by $B(x, r)$ the $k$-ball with center $x$ and radius $r$. Similarly, by $S(x, r)$ we shall denote the $(k-1)$-sphere with center $x$ and radius $r$.

If $v(x)=\left[v_{1}(x), \cdots, v_{k}(x)\right]$ is a Lebesgue measurable vector field defined almost everywhere in $B\left(x_{0}, r_{0}\right)$ (that is each component is defined almost everywhere in $B\left(x_{0}, r_{0}\right)$ and is Lebesgue measurable), we shall say that the approximate divergence of $v$ exists at the point $x_{0}$ and equals $\alpha$ providing the following two facts hold:

(i) There exists a 1-dimensional Lebesgue measurable set $Q$ situated on the positive real axis which has 0 as a point of density from the right such that for $r$ in $\left(0, r_{0}\right) \cap Q$

$$
\int_{S\left(x_{0}, r\right)}\left|v_{j}(x) n_{j}(x)\right| d S(x)<\infty, \quad j=1, \cdots, k,
$$

where $n(x)=\left[n_{1}(x), \cdots, n_{k}(x)\right]$ is the outward pointing unit normal to $S\left(x_{0}, r\right)$ at the point $x$ and $d S(x)$ is the natural $(k-1)$-dimensional volume element on $S\left(x_{0}, r\right)$.

(ii) As $r \rightarrow 0$ through the points of $Q$,

$$
\left|B\left(x_{0}, r\right)\right|^{-1} \int_{S\left(x_{0}, r\right)} v(x) \cdot n(x) d S(x) \rightarrow \alpha
$$

where $\left|B\left(x_{0}, r\right)\right|$ stands for the $k$-volume of $B\left(x_{0}, r\right)$.

If (i) and (ii) hold, we shall henceforth write ap div $v\left(x_{0}\right)=\alpha$.

It is clear that if $v(x)$ is in class $C^{1}$ in $B\left(x_{0}, r_{0}\right)$, then ap $\operatorname{div} v\left(x_{0}\right)$ exists and equals the usual divergence of $v$ evaluated at $x_{0}$.

With an integral lattice point designated by $m$ where $m$ $=\left(m_{1}, \cdots, m_{k}\right)$ and using the notation $(m, x)=m_{1} x_{1}+\cdots+m_{k} x_{k}$ and $|m|=(m, m)^{1 / 2}$, we shall say the $k$-dimensional trigonometric series $\sum a_{m} e^{i(m, x)}$ is in class $D$ if for each $j$ there is a function $v_{j}(x)$ in $L^{1}\left(T_{k}\right)$ which has

$$
\sum^{\prime}-i a_{m} m_{j}|m|^{-2} e^{i(m, x)}
$$

Received by the editors September 11, 1967.

1 This research was sponsored by the Air Force Office of Scientific Research, Office of Aerospace Research, United States Air Force, under AFOSR Grant No. AFAFOSR 694-66. 
as its Fourier series. If this is the case, we shall write

$$
v_{j}(x) \sim \sum^{\prime}-i a_{m} m_{j}|m|^{-2} e^{i(m, x)}, \quad j=1, \cdots, k,
$$

and set

$$
v(x)=\left[v_{1}(x), \cdots, v_{k}(x)\right] .
$$

( $T_{k}$, as usual, designates the $k$-dimensional torus, and $\sum^{\prime}$ the fact that the 0 -lattice point is not to be considered. For multiple trigonometric series, we shall use the notation of [1].)

We shall say that $\sum a_{m} e^{i(m, x)}$ is spherically convergent at the point $x_{0}$ to $\alpha$ if

$$
\lim _{u \rightarrow \infty} \sum_{|m| \leq u} a_{m} e^{i\left(m, x_{0}\right)}=\alpha .
$$

In this paper, we shall establish the following theorem:

THEOREM. Let $\sum a_{m} e^{i(m, x)}$ be a k-dimensional trigonometric series in class $D$ which is spherically convergent at the point $x_{0}$ to $\alpha$ (of finite modulus). Then

$$
\text { ap } \operatorname{div} v\left(x_{0}\right)=\alpha-a_{0},
$$

where $v(x)$ is the vector field defined by (2).

A close look at the definitions given will show that the above theorem is a generalization (and an improvement) of the theorem given in $[2$, p. 324].

2. We now proceed with the proof of the theorem. Without loss of generality, we assume that $x_{0}$ is the origin and that both $a_{0}=0$ and $\alpha=0$.

For each $j$, we are given $v_{j}(x)$ in $L^{1}\left(T_{k}\right)$ satisfying (1).We define the 1-dimensional set $P$ to be

$$
P=\left\{r: \int_{S(0, r)}\left|v_{j}(x)\right| d S(x)<\infty \text { for } j=1, \cdots, k \text { and } 0<r<1\right\} .
$$

Since for each $j, v_{j}(x)$ is also in $L^{1}$ on $B(0,1)$, it follows from Fubini's theorem that $P$ is a Lebesgue measurable set and furthermore that

(3) $\quad \mu(P)=1$ where $\mu$ is 1-dimensional Lebesgue measure.

Next we set $A_{u}(x)=\sum_{|m| \leq u} a_{m} e^{i(m, x)}$. Observing that $d\left[J_{p}(t) t^{-p}\right] / d t$ $=-J_{p+1}(t) t^{-p}$, where $J_{p}(t)$ is the Bessel function of the first kind and $\operatorname{order} p$, and recalling that $a_{0}=0$, we obtain that for $r>0$ 


$$
\begin{aligned}
\sum_{|m| \leq u} a_{m} J_{k / 2}(\mid & m \mid r) /(|m| r)^{k / 2} \\
& =r \int_{0}^{u} A_{t}(0) J_{(k+2) / 2}(t r)(t r)^{-k / 2} d t+A_{u}(0) J_{k / 2}(u r)(u r)^{-k / 2}
\end{aligned}
$$

Since both $J_{k / 2}(u)$ and $J_{(k+2) / 2}(u)$ are $O\left(u^{-1 / 2}\right)$ as $u \rightarrow \infty$ and since by assumption $A_{u}(0)=o(1)$ as $u \rightarrow \infty$, we conclude that

$$
\begin{aligned}
& \lim _{u \rightarrow \infty} \sum_{|m| \leq u} a_{m} J_{k / 2}(|m| r)(|m| r)^{-k / 2} \\
& =r \int_{0}^{\infty} A_{u}(0) J_{(k+2) / 2}(u r)(u r)^{-k / 2} d u .
\end{aligned}
$$

The integral on the right side of the equality in (4) is absolutely convergent for every $r>0$; consequently, the limit on the left side of the equality is finite for every $r>0$. We obtain, therefore, that

$$
\lim _{t \rightarrow 0} \sum_{m} a_{m} J_{k / 2}(|m| r)(|m| r)^{-k / 2} e^{-|m| t}
$$

$$
=\lim _{u \rightarrow \infty} \sum_{|m| \leq u} a_{m} J_{k / 2}(|m| r)(|m| r)^{-k / 2} \quad \text { for } r>0 .
$$

Next, we set for $t>0$

$$
v_{j}(x, t)=\sum_{m}^{\prime}-i a_{m} m_{j}|m|^{-2} e^{i(m, x)-|m| t}
$$

and

$$
v(x, t)=\left[v_{1}(x, t), \cdots, v_{k}(x, t)\right] .
$$

From a familiar computation involving Bessel functions, we obtain

$$
\begin{aligned}
& |B(0, r)|^{-1} \int_{S(0, r)} v(x, t) \cdot n(x) d S(x) \\
= & \gamma_{k} \sum_{m} a_{m} J_{k / 2}(|m| r)(|m| r)^{-k / 2} e^{-|m| t},
\end{aligned}
$$

where $\gamma_{k}$ is a positive constant depending on $k$.

From (4), (5), and (8), it follows that, for $0<r<1, g(r)$ exists and is finite where $g(r)$ is defined by the following limit

$$
g(r)=\lim _{t \rightarrow 0} \int_{S(0, r)} v(x, t) \cdot n(x) d S(x) .
$$


We note that for $t>0, v(x, t)$ is a continuous vector-valued function in $x$; so $g(r)$ is a Borel measurable function of $r$ on the interval $(0,1)$. We recall that $v_{j}(x)$ is a function in $L^{1}\left(T_{k}\right)$ satisfying (1). It follows therefore from (6) and well-known facts concerning multiple Fourier series, e.g. $[1,(17)$, p. 56$]$, that

$$
\int_{T_{k}}\left|v_{j}(x)-v_{j}(x, t)\right| d x \rightarrow 0 \quad \text { as } t \rightarrow 0 \quad \text { for } j=1, \cdots, k \text {. }
$$

Using these facts, we next show

$$
\int_{0}^{1}\left|\int_{S(0, r)} v(x) \cdot n(x) d S(x)-g(r)\right| d r=0 .
$$

We do this by observing that, by Fatou's lemma and (9), the expression on the left side of (11) is majorized by

$$
\liminf _{t \rightarrow 0} \int_{0}^{1}\left|\int_{S(0, r)}[v(x)-v(x, t)] \cdot n(x) d S(x)\right| d r .
$$

But this expression in turn is majorized by

$$
\liminf _{t \rightarrow 0} \sum_{j=1}^{k} \int_{B(0,1)}\left|v_{j}(x)-v_{j}(x, t)\right| d x
$$

which is 0 by (10); and (11) is established.

Next, we observe from (11) and (3) that the Lebesgue measurable set $Q$ defined by

$$
Q=\left\{r: \int_{S(0, r)} v(x) \cdot n(x) d S(x)=g(r) \text { and } \quad r \text { in } P\right\}
$$

is such that

$$
\mu(Q)=1 .
$$

In order to complete the proof of the theorem, we need the following fact which we shall establish next:

$$
\int_{\rho}^{2 \rho}|g(r)| r^{-k} d r=o(\rho) \quad \text { as } \rho \rightarrow 0 .
$$

To establish (14), we observe from (4), (5), (8), and (9) that $g(r) r^{-k}$ is a positive constant multiple of $r \int_{0}^{\infty} A_{u}(0) J_{(k+2) / 2}(u r)(u r)^{-k / 2} d u$. By assumption, $A_{u}(0)=o(1)$ as $u \rightarrow \infty$. Consequently, for $0<2 \rho<1$, 


$$
\begin{aligned}
\int_{\rho}^{2 \rho} g(r) r^{-k} d r= & \int_{0}^{1 / \rho} o(1)\left\{\int_{\rho}^{2 \rho} r\left|J_{(k+2) / 2}(u r)\right|(u r)^{-k / 2} d r\right\} d u \\
& +\int_{1 / \rho}^{\infty} o(1)\left\{\int_{\rho}^{2 \rho} r\left|J_{(k+2) / 2}(u r)\right|(u r)^{-k / 2} d r\right\} d u .
\end{aligned}
$$

Calling the first expression on the right side of the equality in (15) $I_{\rho}^{\prime}$ and the second $I_{\rho}^{\prime \prime}$ and using the familiar facts that there are constants $K^{\prime}$ and $K^{\prime \prime}$ such that $\left|J_{(k+2) / 2}(t)\right| \leqq K^{\prime} t^{(k+2) / 2}$ for $0<t \leqq 2$ and $\left|J_{(k+2) / 2}(t)\right| \leqq K^{\prime \prime} t^{-1 / 2}$ for $1 \leqq t<\infty$, we see in turn that as $\rho \rightarrow 0$,

$$
I_{\rho}^{\prime \prime} \leqq \int_{0}^{1 / \rho} o(1)\left[\int_{\rho}^{2 \rho} r d r\right] d u=o(\rho)
$$

and

$$
I_{\rho}^{\prime \prime} \leqq \int_{1 / \rho}^{\infty} o(1)\left[\int_{\rho}^{2 \rho} r^{-(k-1) / 2} d r\right] u^{-(k+1) / 2} d u=o(\rho) ;
$$

(14) is consequently established.

We now proceed along the lines given in $[2$, p. 324]. From (14) it follows that

$$
\int_{2^{-(n+1)}}^{2^{-n}}|g(r)||B(0, r)|^{-1} d r=\epsilon_{n} 2^{-n} \quad \text { for } n=1,2, \cdots
$$

where $\lim _{n \rightarrow \infty} \epsilon_{n}=0$.

For $n=1,2, \cdots$, we define the set $E_{n}$ to be

(16) $E_{n}=\left\{r:|g(r)||B(0, r)|^{-1} \geqq \underset{n}{\epsilon^{1 / 2}}, r\right.$ in $Q$, and $\left.2^{-(n+1)} \leqq r \leqq 2^{-n}\right\}$.

Then it follows from $\left(15^{\prime}\right)$ that

$$
\mu\left(E_{n}\right) \leqq \epsilon_{n}^{1 / 2} 2^{-n} .
$$

We set $E=\bigcup_{n=1}^{\infty} E_{n}$ and observe from (17) that $\mu\left[E \cap\left(0,2^{-j}\right)\right] 2^{j} \rightarrow 0$ as $j \rightarrow \infty$. But then (13) implies that

$$
Q-E \text { has } 0 \text { as a point of density from the right. }
$$

Recalling the definition of $P$, we obtain from (12) that

$$
\int_{S(0, r)}\left|v_{j}(x) n_{j}(x)\right| d S(x)<\infty
$$

$$
\text { for } r \text { in } Q-E \text { and } j=1, \cdots, k \text {. }
$$


Next, using (12) in conjunction with (16), we obtain that

$$
|B(0, r)|^{-1} \int_{S(0, r)} v(x) \cdot n(x) d S \rightarrow 0
$$

as $r \rightarrow 0$ through the points of $Q-E$.

But (18), (19), and (20) imply that (i) and (ii) hold with $Q$ replaced by $Q-E$ and $\alpha=0$. We conclude that ap $\operatorname{div} v(0)=0$, and the proof to the theorem is complete.

In closing we point out that for $k \geqq 4$ spherical convergence in the above theorem can be replaced by higher orders of Bochner-Riesz summability [1, p. 49].

\section{BIBLIOGRAPHY}

1. V. L. Shapiro, Fourier series in several variables, Bull. Amer. Math. Soc. 70 (1964), 48-93.

2. A. Zygmund, Trigonometric series, Vol. I, Cambridge Univ. Press, Cambridge, 1959.

University of California, Riverside 